\title{
Anne Eydoux, Bernard Gomel (coord.), Apprendre (de l'échec) du RSA. La solidarité active en question
} Rueil-Malmaison, Éditions Liaisons, coll. « Liaisons sociales », 2014

\section{Cécile Lefèvre}

\section{OpenEdition}

\section{Journals}

Édition électronique

URL : http://journals.openedition.org/travailemploi/6933

DOI : 10.4000/travailemploi.6933

ISSN : $1775-416 \mathrm{X}$

Éditeur

DARES - Ministère du Travail

\section{Édition imprimée}

Date de publication : 1 janvier 2016

Pagination : 200-204

ISSN : 0224-4365

\section{Référence électronique}

Cécile Lefèvre, « Anne Eydoux, Bernard Gomel (coord.), Apprendre (de l'échec) du RSA. La solidarité active en question », Travail et Emploi [En ligne], 145 I janvier-mars 2016, mis en ligne le 01 janvier 2016, consulté le 25 septembre 2020. URL : http://journals.openedition.org/travailemploi/6933 ; DOI : https://doi.org/10.4000/travailemploi.6933 


\title{
Apprendre (de l'échec) du RSA. La solidarité active en question
}

\author{
Anne Eydoux, Bernard Gomel (coord.) \\ Rueil-Malmaison, Éditions Liaisons, coll. « Liaisons sociales », 274 p.
}

\section{Lu par Cécile Lefèvre*}

Paru en 2014, l'ouvrage réunit les contributions de dix-huit chercheurs ou acteurs des politiques sociales. Il échappe à l'écueil le plus courant des ouvrages collectifs, celui du kaléidoscope. Sa ligne directrice est en effet clairement énoncée par ses deux coordinateurs, Anne Eydoux et Bernard Gomel, du Centre d'études de l'emploi, auteurs d'une moitié environ des contributions. Leur objectif est de dresser la genèse et le bilan des cinq premières années de la mise en œuvre du Revenu de solidarité active (RSA). Et la conclusion est limpide : il s'agit d'un échec - échec multiforme même, que les contributions explicitent de manière détaillée sous différents angles. Cet échec s'expliquerait en partie par l'erreur originelle de la réforme : pensant innover dans le domaine de « l'activation » de la solidarité, ses initiateurs ont fait étroitement dépendre un dispositif de protection sociale de comportements d'emploi et du fonctionnement du marché du travail.

L'ouvrage se lit de manière très fluide et agréable en raison de sa structuration thématique précisément annoncée, de chapitres introductifs et problématisés en début de chacune des cinq parties, et de ses contributions courtes et précises. Ce souci de clarté pédagogique est une grande qualité et participe à la force de conviction qui se dégage de l'ouvrage.

Les contributeurs, majoritairement sociologues et/ou économistes sont tous des spécialistes des politiques sociales et de l'emploi : fins connaisseurs du RSA, ils proposent aussi de le mettre en perspective, en l'inscrivant dans l'histoire et dans l'analyse plus globale des réformes des politiques sociales en France. Quelques contributeurs participent également en tant qu'acteurs institutionnels à la mise en œuvre de ces politiques (que ce soit au niveau d'un conseil départemental, d'une des caisses d'allocations familiales ou de l'Agence nouvelle des solidarités actives [Ansa]), apportant ainsi un point de vue original sur la mise en place concrète du RSA.

\footnotetext{
* Centre de recherche sur les liens sociaux (Cerlis), université Paris Descartes, Sorbonne Paris Cité.
} 
La première partie, intitulée «La "grande transformation" des politiques de lutte contre la pauvreté » revient sur l'origine de la création du $\mathrm{RSA}^{1}$, à la fois sur le plan légal et d'un point de vue conceptuel. Ses principes sont esquissés dès 2005, dans un rapport de Martin Hirsch sur la pauvreté des familles. Le RSA, qui s'est substitué ensuite à la fois au Revenu minimum d'insertion (RMI) et à l'Allocation parent isolé (API), entendait infléchir les politiques de solidarité et d'aide sociale en France en les soumettant plus fortement et plus explicitement à l'impératif d'insertion professionnelle et de retour à l'emploi. Selon les auteurs, ce choix principiel serait la cause de l'échec du RSA, qui ne doit donc pas être mis sur le compte de difficultés externes et conjoncturelles de l'économie française. Cette première partie de l'ouvrage souligne également que l'objectif d'activation n'est pas une complète innovation. Une bonne part des contrats d'insertion conclus avec les bénéficiaires du RMI visait déjà l'insertion professionnelle. Cet objectif s'inscrit en fait dans une tendance de fond dans la mesure où la plupart des pays européens ont, depuis les années 1990, réformé de manière articulée leurs systèmes d'indemnisation du chômage, leurs minima sociaux et leur fiscalité. Ils ont ainsi instauré des « régimes » d'activation variés que recouvre la notion «d'État social actif» ou «d'État d'investissement social ». Dans ce cadre, les auteurs regrettent que le ciblage du RSA sur la population d'âge actif ait conduit à négliger l'autre objectif initial de la réforme, à savoir la lutte contre la pauvreté des enfants et des jeunes. Les critères d'éligibilité au RSA, ouvert en principe aux moins de 25 ans (ce qui est a priori une avancée, répondant à l'une des principales critiques faites au RMI), s'avèrent de fait quasiment impossibles à réunir pour ces jeunes. Les auteurs relèvent enfin que la phase et la procédure d'évaluation, qui devaient contribuer à tester et à affiner de manière rigoureuse le dispositif du RSA, et ainsi contribuer à sa légitimité, ont finalement été très rapidement conclues et seulement partiellement utilisées ; la dynamique envisagée d'évaluation-adaptation permanente par expérimentation, dont l'importance avait été initialement soulignée, a donc été écartée.

La deuxième partie est consacrée à l'examen de l'objectif premier assigné au RSA : « agir sur le marché du travail ». Le bilan serait (ou est, selon les auteurs) de ce point de vue très décevant. Certes, le dispositif n'aurait pas entraîné les effets pervers redoutés : les employeurs n'auraient guère instrumentalisé le RSA pour tirer les salaires vers le bas, mais ce serait essentiellement par méconnaissance du dispositif, qui reste à leurs yeux une allocation relevant avant tout du domaine de l'assistance sociale aux plus démunis. Mais le RSA n'aurait pas non plus entraîné les effets positifs escomptés de retour à l'emploi et d'incitation à travailler. Dans bien des cas, les allocataires se caractériseraient par un « éloignement de l'emploi ${ }^{2} »$ qu'une simple incitation monétaire ne saurait résoudre, tandis que les personnes les plus proches de l'emploi ne feraient guère valoir leurs droits au RSA activité. Certains considèrent

1. En décembre 2008, à la suite d'une phase d'expérimentations.

2. Cet éloignement s'expliquerait par une formation insuffisante ou très inadaptée à l'offre d'emploi, un mauvais état de santé, des problèmes de garde d'enfants, de transports, etc., et le manque de création d'emplois. 
le dispositif comme stigmatisant, d'autres comme complexe à comprendre, et générant, par son fonctionnement trimestriel, des décalages entre déclarations et versements de la prestation, ainsi que des indus difficiles à gérer. Finalement, le RSA modifierait bien peu les déséquilibres du marché du travail ; il contribuerait même à leur pérennité et «participerait à (leur) régulation» (p. 74).

La troisième partie intitulée "Redistribution, chronique d'un échec annoncé » revient sur le second objectif majeur du RSA, cette fois en tant que minimum social : la lutte contre la pauvreté. Selon l'ouvrage, le bilan serait également négatif en la matière. Tout d'abord, le RSA socle consacrerait le mouvement de diminution de la valeur du minimum social relativement au salaire minimum interprofessionnel de croissance (Smic) (de $50 \%$ du Smic en 2001 pour le RMI à $43 \%$ en 2013 pour le RSA). Par ailleurs, la création de deux composantes du RSA (socle et activité), loin de sécuriser les personnes, aurait été contre-productive pour plusieurs raisons. Le montant du RSA activité varie en fonction des fluctuations du revenu du travail ; cette labilité, qui intervient avec décalage en raison des déclarations trimestrielles de revenus, créerait un sentiment d'instabilité. De plus, le RSA socle est calculé en fonction du revenu du ménage et suivant une logique de besoin, tandis que le RSA activité dépend du comportement d'activité individuel et correspond à une logique de complément incitatif de revenu. Mêler les deux peut sembler contradictoire et est source de confusion. De fait, l'importance inattendue et durable du non-recours au RSA est un signe fort de dysfonctionnement du dispositif, et conduit à un déficit de redistribution. En 2011, on estimait que $49 \%$ des bénéficiaires potentiels n'auraient pas fait valoir leurs droits, ce taux s'élevant à $68 \%$ dans le cas du seul RSA activité, si bien que les auteurs concluent que «le RSA passe largement à côté de son public cible » (p. 146). Enfin, point moins connu et mis en lumière par l'ouvrage, la réforme du RSA a été l'occasion d'un durcissement très net des conditions d'accès au droit pour les étrangers, qui doivent désormais justifier d'une résidence en France d'au moins cinq années, ainsi que d'une situation de séjour régulière avec autorisation de travail.

La quatrième partie portant sur « Les acteurs et la gouvernance du RSA » analyse la dimension institutionnelle du dispositif. Sa mise en place a initié un changement marquant, en faisant de Pôle emploi un de ses acteurs principaux, au même titre que les caisses d'allocations familiales (CAF) et les conseils départementaux. Ce montage institutionnel a pour conséquence une segmentation de la population des bénéficiaires. En effet, ceux déjà inscrits à Pôle emploi ou proches de l'emploi sont suivis par l'opérateur public tandis que les autres, relevant davantage de problématiques « d'insertion sociale », sont accompagnés de manière très diverse suivant les territoires : par la CAF ou par différentes associations mandatées par les conseils départementaux. Cette catégorisation des publics et cette multiplication des acteurs sont contraires au sens initial de la réforme, qui souhaitait ne plus dissocier politiques d'aide sociale et d'accompagnement vers l'emploi. Or elles ont accentué les différences de situations et de traitement des potentiels bénéficiaires suivant les départements, ce qui était l'un des points faibles déjà identifiés du RMI. De plus, les modes de financement amplifient 
ces effets de disparités territoriales et de complexification du réseau d'acteurs dans la mesure où le RSA activité est financé par le Fonds national de solidarité active (FNSA) et l'État, tandis que le RSA socle relève des départements.

La cinquième partie propose de détailler «Les mises en œuvre de l'accompagnement », idée phare de la réforme du RSA. Elle s'appuie sur des enquêtes et études de $\operatorname{cas}^{3}$ et avance des conclusions nuancées. Alors que la réforme a inscrit Pôle emploi au cœur du dispositif et de l'accompagnement qu'il prévoit, la traduction dans les faits a été variable selon les départements. L'évaluation de l'employabilité du bénéficiaire et de son projet professionnel lors de l'entrée dans le RSA s'est avérée difficile à mettre en œuvre, d'autant que les critères sont fluctuants d'un endroit à l'autre. De plus, l'accompagnement a dû être déployé sans que des moyens supplémentaires en cadres territoriaux ou travailleurs sociaux soient attribués. Une innovation intéressante du RSA - l'inscription dans la loi de la participation des bénéficiaires à la gestion du dispositif dans le cadre de l'Agence nouvelle des solidarités actives (Ansa) - fait l'objet du dernier chapitre et d'une appréciation plutôt positive mais, comme le regrette le Mouvement national des chômeurs et des précaires (MNCP), il s'agit d'une participation à titre individuel, sans représentation collective.

L'ouvrage conclut fort logiquement en proposant de « découpler la solidarité de l'activation », d'élargir le champ du RSA aux jeunes et aux étrangers en situation régulière et d'augmenter le montant du RSA socle. Rendre le travail plus rémunérateur devrait passer par une revalorisation du Smic et la lutte contre les contrats, durées et conditions de travail qui entretiennent la précarité plutôt que par les incitations financières fragiles et fluctuantes proposées par le RSA actuel. Certains des contributeurs suggèrent également de sortir d'une logique «ménage » et d'individualiser, ou de personnaliser davantage le droit social à un revenu minimum.

On l'aura compris, il s'agit au final d'un ouvrage collectif précieux, défendant une thèse, engagé et pédagogique, proposant un bilan d'étape argumenté du RSA.

Malgré notre enthousiasme, nous nous proposons de soulever quelques points de critiques ou plutôt de souhaits ou de prolongements.

Si l'ouvrage s'appuie à plusieurs reprises sur des enquêtes et des études de cas, il s'agit le plus souvent de données administratives agrégées, d'un examen global d'une situation, d'analyses secondaires. Ce n'était certes pas le but de l'ouvrage, mais le lecteur reste un peu sur sa faim s'il cherche à mieux comprendre et mieux connaitre de manière plus concrète les parcours des allocataires et leurs perceptions du dispositif.

Les études de cas mobilisées dans l'ouvrage sont presque toutes situées en Îlede-France ; quelques exemples plus concrets dans d'autres régions et départements seraient utiles et permettraient de mieux mettre en lumière cette diversité territoriale et ce jeu d'acteurs, énoncés et analysés dans l'ouvrage mais peu « mis en scène ».

3. Sont ainsi étudiés la mise en œuvre du RSA par la ville de Paris ainsi que l'accompagnement de certains groupes professionnels comme les artistes ou l'implication des bénéficiaires dans la gestion du dispositif. 
La question du financement et les discussions récentes sur l'évolution du dispositif auraient pu être davantage explorées. On a observé en effet les difficultés croissantes de certains départements à financer le RSA socle devant l'augmentation des situations de grande pauvreté. Certains en appellent à un changement des règles de financement et à plus de solidarité collective nationale ; d'autres seraient tentés par un durcissement des règles d'accès, voire par l'introduction de conditions supplémentaires pour l'ouverture des droits, dans une logique de workfare (aide sociale conditionnée au fait de travailler).

Développer davantage ces questions viendrait d'ailleurs à l'appui des idées principales de l'ouvrage. 